\title{
7 Evaluating Current Dental Imaging Modalities for Human Bite Mark
}

IJCRR
ection: Healthcare

Sci. Journal Impact

Factor: $6.1(2018)$

ICV: 90.90 (2018)

(c) (7) (5)

Copyright@IJCRR

\section{Rameswari Poornima Janardanan ${ }^{1,2}$, Rajasvaran Logeswaran²}

'Riyadh Elm University, Riyadh, Kingdom of Saudi Arabia; ${ }^{2}$ Asia Pacific University of Technology and Innovation, Kuala Lumpur, Malaysia.

\section{ABSTRACT}

Objective: This study evaluates the image acquisition efficacy of current available dental imaging modalities for human bite marks in forensic odontology. Dental casts were collected retrospectively from Riyadh dental Hospital.

Methods: Human bite marks were registered on different materials like pink wax, aluwax and Polyvinyl siloxane. These study casts with their corresponding bite marks were imaged and categorized into: (1) 2D imaging using digital cameras following the American Board of Forensic Odontology guidelines; (2) 2D CBCT imaging with Cone-beam computed tomography (CBCT); and (3) 3D imaging using CAD/CAM and CBCT. Sensitivity, specificity and receiver operating characteristics (ROC) curves were traced for observers in all the approach. Paired t-test and kapa tests were utilized for the inter-observer consistency. Each modality had a significant difference under the ROC plotted.

Results: The study observed that 3D CBCT imaging in comparison with the other imaging modalities had a marginally higher value of $P=0.0002$. The kappa method for the inter-observer agreement was used to confirm this observation.

Conclusion: This study clearly shows the advantage of 3D imaging using CBCT in human bite mark imaging. Based on the results, a 3D forensic odontological image database collection with a gold standard is the future potential of this study.

Key Words: Technical evaluation, CBCT, ROC, CAD, CAM, Human bite marks

\section{INTRODUCTION}

Digital human bite mark image acquisition and analysis is a predominant feature in both forensic and biometric applications. Recently there has been a revolution in dental imaging technologies. The field of forensic odontology has benefited from the newer digital imaging modalities, especially with the realization of low dose 3D computed Tomography (CT), along with much such innovative imaging techniques. ${ }^{1}$ These state of the art innovations in imaging in which digital data can be combined and manipulated can be utilized by experts to easily access and assess 3D simulations on the screen to accurately plan and follow up their clinical decisions over time. $^{2}$

Bite mark injuries had always been of interest to forensic and judiciary system. ${ }^{3}$ Though human bite mark pattern comparisons have been a forensic tool and are accepted in the court of law, it always has been in debate due to the intricate nature of the human skin in retaining the bite pattern. ${ }^{4}$ From the different types of imaging available, the 2D digital camera along with photo editing software has the currently accepted modality for bite mark image acquisition. All branches and application of dentistry have witnessed in recent decades the prodigious development of different imaging modalities. With these advances, the need for more precise diagnostic tools, especially imaging methods, have become mandatory. ${ }^{5}$ Correct use of newer radiographic techniques, where indicated, allows for better precision of forensic identifications. However, it is imperative to utilize these imaging modalities with the proper knowledge to extract the best advantage of these in the best practice of forensics. Since its introduction in CBCT has gained its notable place as a radiographic diagnostic tool among clinicians. ${ }^{6}$ This study evaluates the imaging accuracy and acceptance of the current available dental imaging modalities in imaging bite marks.

\section{Corresponding Author:}

Rameswari Poornima Janardanan, Riyadh Elm University, Riyadh, Kingdom of Saudi Arabia. Email: ahlambaha@hotmail.com

ISSN: 2231-2196 (Print)

Received: 20.07 .2020
ISSN: 0975-5241 (Online)

Revised: 12.09 .2020
Accepted: 20.10 .2020
Published: 12.11 .2020 


\section{MATERIALS AND METHODS}

A total of 95 dental casts were collected retrospectively from Riyadh Elm University Hospital. Bite marks were registered using different materials (Pink wax, aluwax and Polyvinyl siloxane). The study casts with their corresponding bite marks were imaged into 3 categories, as shown in Table 1.

Table 1: Categories of the imaging modalities evaluated in the study.

\begin{tabular}{|c|c|c|c|}
\hline \multicolumn{2}{|l|}{ 2D imaging } & \multicolumn{2}{|l|}{ 3D imaging } \\
\hline Photographs & СВCТ & CAD/CAM & СВСТ \\
\hline $\begin{array}{l}\text { Photographed using digital cameras } \\
\text { canon EOS } 700 \text { DFS } 18-55 \mathrm{~mm} \text { and } \\
\text { Sony Cyber-shot DCS-RX10o VI follow- } \\
\text { ing the ABFO guidelines. }\end{array}$ & $\begin{array}{l}\text { 2D Scans of the casts } \\
\text { were taken using CBCT, } \\
\text { Galileos, Sirona, Ger- } \\
\text { many. }{ }^{7}\end{array}$ & $\begin{array}{l}\text { 3D Scans of the casts were } \\
\text { taken using CAD/CAM, } \\
\text { CEREC 3D, Sirona Dental } \\
\text { Systems Germany. }{ }^{8}\end{array}$ & $\begin{array}{l}\text { 3D Scans of the casts were } \\
\text { taken using CBCT, Galileos, } \\
\text { Sirona, Germany. }\end{array}$ \\
\hline
\end{tabular}

\section{Data collection}

The present study was carried out in the Riyadh Elm University (REU), Riyadh, Kingdom of Saudi Arabia, after being approved by the institutional review board of REU. Experimental bite marks were obtained from a total of 95 dental casts, collected retrospectively from REU dental hospital. The inclusion creation for casts selection was the presence of all incisors and canine teeth to register the typical possible bite mark injury. The exclusion criterion in this study was missing and mobile anterior teeth, and diseases of teeth such as severe attrition of anterior teeth This was done to eliminate the possibility of error in the impressions taken due to the underlying etiology.

\section{Data preparation}

The dental casts were made using alginate impression material (MA-A $7100^{\circledR}$, Major Dental, Italy) prepared according to the manufacturer's specifications and in conjunction with accepted dental laboratory techniques. From these casts, bite marks were registered using different materials, specifically, pink wax, aluwax and polyvinyl siloxane. This study casts with their corresponding bite marks were imaged into 3 categories. Both the dental casts and bite registrations were scanned using 3 types of dental imaging modalities.

\section{D Photographic Image acquisition}

The dental casts, along with the bite registrations were photographed as in Figure 1(a) and (b), with Canon EOS 700D EFS $18-55 \mathrm{~mm}$ and Sony Cyber-shot DCS-RX100 VI, respectively. The ABFO No. 2 scale was used while imaging. The graphic program Adobe Photoshop ${ }^{\circledR}$ was used in tracing the incisal edge contours and outlines from the casts. The metric features of the anterior teeth arches and the individual teeth were sent to expert oral radiologists to be accepted and analyzed for bite marks comparisons.
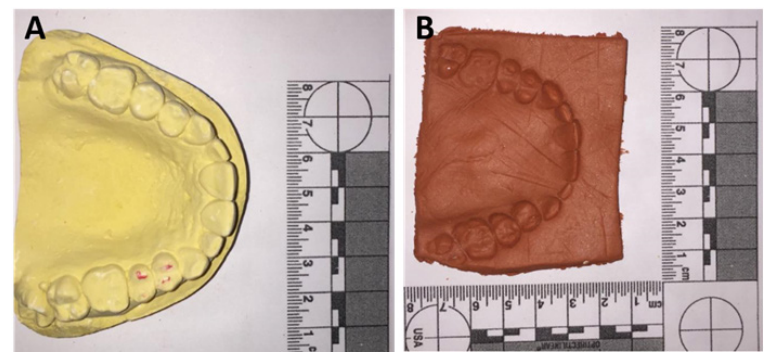

Figure 1: 2D photographs of the dental cast $(A)$ and bite mark registration $(B)$.

\section{D CAD-CAM image acquisition}

The study casts and the registered bitemarks from it were imaged using the intraoral digital scanner CAD/CAM (CEREC 3D, Sirona Dental Systems GmbH, Germany) and with CEREC Software ${ }^{\circledR}$, which is the software for digital acquisition and $3 \mathrm{D}$ design. It produced an image coloured by the variation of tissue color, as seen in Figure 2.

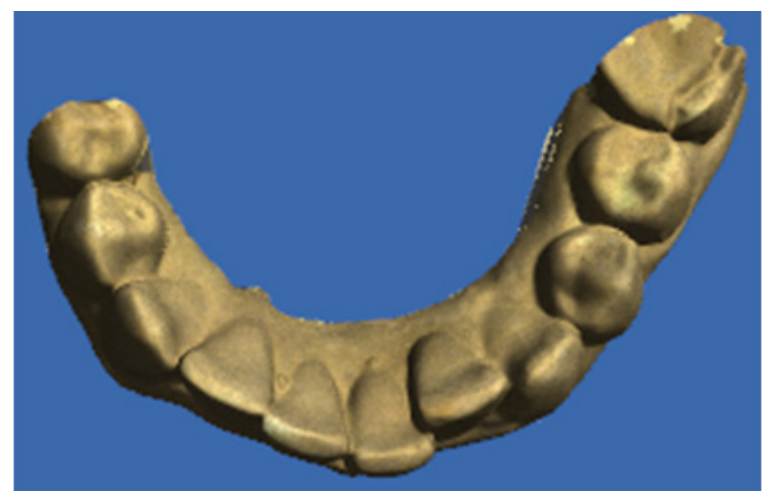

Figure 2: 3D image of dental cast from CAD/CAM

\section{D and 3D CBCT image acquisition}

The $2 \mathrm{D}$ radiographic and $3 \mathrm{D}$ imaging capabilities of the CBCT equipment Galileos, Sirona, Germany was utilized. The bite marks from the dental casts were subjected to scan using the Sirona Gallelios CBCT machine. The param- 
eters for exposure were $84 \mathrm{kVp}, 14 \mathrm{~mA}$, and 12 seconds, $401 \times 401 \times 401$ pixels matrix, a voxel size of $200 \mu \mathrm{m}$, and a slice thickness of $1 \mathrm{~mm}$. The study casts were also scanned individually with the same parameters as in the bite marks registered, and volumetric data were collected. Both the dental casts and bite registrations were analyzed through the tomographic slices and the $3 \mathrm{D}$ reconstructions.

SIDEXIS $4^{\circledR}$ software was used for analyzing the DICOM file which constituted the volumetric data of both cats and the bite marks. These DICOM images of bite mark registrations were constructed as in Figure 3 and were analyzed by four observers at different periods. The raters were all oral radiology specialists, and they independently evaluated the images in two different viewing sessions. The degree of confidence in the given images was marked by these experts. Their confidence in detecting the clarity and details of a bite mark in the given 2D and 3D images were rated using a fivepoint Likert scale, as given in Table 2.
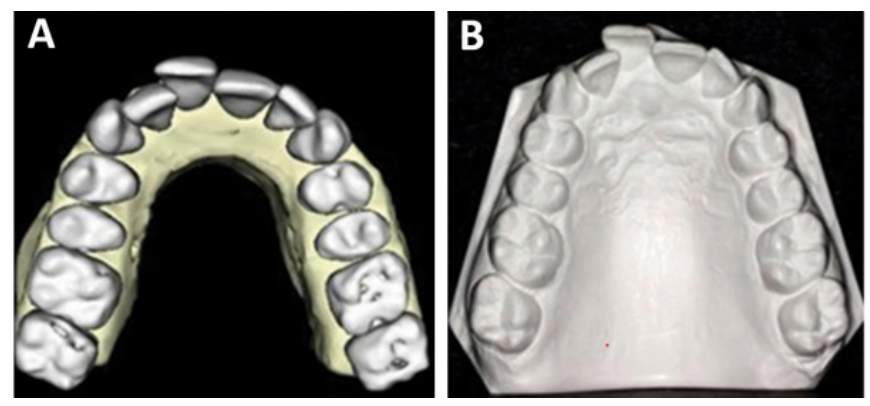

Figure 3: 3D image of the dental cast from CBCT scans $(A)$ of the actual dental cast used (B).

Table 2: The 5-point scale used for evaluation of clarity and bite mark detail in the $2 \mathrm{D}$ and ${ }_{3} \mathrm{D}$ images.

\begin{tabular}{ll} 
Scale & Description \\
1 & Accurate for further analysis \\
2 & Good for further analysis \\
3 & Average for further analysis \\
4 & Barely enough for further analysis \\
5 & Not fit for further analysis \\
\hline
\end{tabular}

\section{Statistical analysis}

Statistical Package for Social Sciences (SPSS) version 21.0 was used in all the statistical observations in this study. The diagnostic accuracy of the three modalities (Photography, $\mathrm{CAD} / \mathrm{CAM}$ and $\mathrm{CBCT}$ ) was evaluated using the area under the receiver operating characteristic (ROC) and sensitivity and specificity values using analysis of variance. Interobserver agreement was analyzed by the kappa ( $\kappa)$ statistic.

\section{RESULTS AND DISCUSSION}

It was seen $\mathrm{CBCT}$ had a notably higher value than the other modalities with a remarkable difference in the areas under the ROC, as shown in Figure 4. All the imaging modalities had a similar result in specificity and table 3 shows the interrater compatibility using kappa statistics in assessing ease and clarity of bite marks from the images, by modality. 3D CBCT at $95 \%$ confidence interval(CI) had an impressive sensitivity compared to te other modalities for imaging.

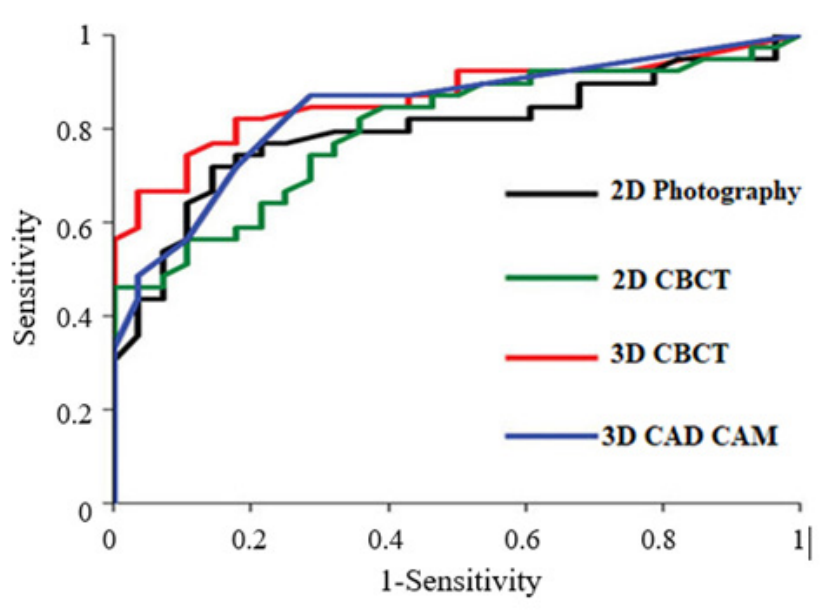

Figure 4: Receiver operating characteristic (ROC) curves for all 3 modalities

Table 3: Interrater agreement in assessing ease and clarity of bite marks from the images, by modality.

\begin{tabular}{lc} 
Imaging approach & $\kappa\left(\begin{array}{c}5 \text { category scale }) \\
(95 \% \mathrm{CI})\end{array}\right.$ \\
\hline $\mathrm{CBCT}_{3} \mathrm{D}$ imaging & $0.61(0.570 .65)$ \\
$\mathrm{CAD} \mathrm{CAM} 3 \mathrm{D}^{\mathrm{C}}$ & $0.60(0.550 .64)$ \\
$\mathrm{CBCT} 2 \mathrm{D}$ imaging & $0.27(0.230 .31)$ \\
$2 \mathrm{D}$ photography & $0.32(0.280 .35)$ \\
\hline
\end{tabular}

The 3D imaging function of CBCT proved to be marginally sensitive than the other two imaging modalities, as can be seen in Table 4. It was seen that was room for $31 \%$ false negatives where a bite mark could be analyzed, even with a sensitivity of $69 \%$ in the case of CBCT. This points out that to improve the clarity of the bite mark details, it is necessary to refine the image acquisition and method of $3 \mathrm{D}$ CBCT. 
Table 4: The area under receiver operating characteristic (ROC) curve, average sensitivity and specificity by modality

\begin{tabular}{|c|c|c|c|}
\hline Imaging approach & ROC Area (95\% CI) & $\begin{array}{c}\text { Sensitivity (95\% CI) } \\
(\%)\end{array}$ & $\begin{array}{c}\text { Specificity }(95 \% \text { CI }) \\
(\%)\end{array}$ \\
\hline $\mathrm{CBCT}_{3} \mathrm{D}$ imaging & $\begin{array}{c}0.87 \\
(0.79-0.89)\end{array}$ & $\begin{array}{c}69.44 \\
(60.41-78.47)\end{array}$ & $\begin{array}{c}92.35 \\
(82.22-100.0)\end{array}$ \\
\hline $\mathrm{CAD} \mathrm{CAM}_{3} \mathrm{D}$ & $\begin{array}{c}0.85 \\
(0.77-0.88)\end{array}$ & $\begin{array}{c}68.23 \\
(65.11-74 \cdot 36)\end{array}$ & $\begin{array}{c}91.2 \\
(90.06-98.18)\end{array}$ \\
\hline $\mathrm{CBCT} 2 \mathrm{D}$ imaging & $\begin{array}{c}0.71 \\
(0.69-0.74)\end{array}$ & $\begin{array}{c}51.85 \\
(42.8260 .88)\end{array}$ & $\begin{array}{c}83.65 \\
\left(74.5^{2-92.78)}\right.\end{array}$ \\
\hline \multirow[t]{2}{*}{ 2D photography } & $\begin{array}{c}0.71 \\
(0.68-0.73)\end{array}$ & $\begin{array}{c}51.54 \\
(42.5160 .57)\end{array}$ & $\begin{array}{c}79.17 \\
(70.04-88.30)\end{array}$ \\
\hline & $\mathrm{P}=0.0002$ & $\mathrm{P}=0.0174$ & $\mathrm{P}=0.1573$ \\
\hline
\end{tabular}

\section{CONCLUSION}

This study shows the advantage of 3D imaging using CBCT in human bite mark imaging. It is also seen from this study that 3D CADCAM is a very promising modality to explore in the bite mark identity studies. These modalities if explored and utilized well could be of greater assistance in legal and judicial aspects. In this study, it was shown that CBCT was efficient in yielding images with the finer level of detail needed to detect and compare human bite marks. CBCT imaging was shown to be more resourceful in bitemark imaging and thus if used wisely, CBCT 3D imaging could replace the conventional 2D bite mark imaging as an efficient forensic tool.

\section{ACKNOWLEDGEMENTS}

The authors like to acknowledge the support from the Research department of REU and the kind mentor, Dr. Abdullah R AlShammery, Chairman, Board of Trustees, REU. Riyadh, Kingdom of Saudi Arabia.

\section{Conflict of Interest}

The authors involved in the current study does not declare any competing conflict of interest.

\section{Funding and Sponsorship}

No fund or sponsorship in any form was obtained from any organization for carrying out this research work.

\section{REFERENCES}

1. Todd R. Dental imaging - 2 D to 3 D: a historic, current, and future view of projection radiography. Endodontic Topics. 2014 Nov;31(1):36-52.

2. Rekow ED. Digital dentistry: The new state of the art-Is it disruptive or destructive?. Dental Materials. 2020 Jan 1;36(1):9-24.

3. David TJ, Lewis JM. Patterned Injury Analysis and Bitemark Comparison. InForensic odontology 2018 Jan 1 (pp. 173-206). Academic Press.

4. de Boer HH, Blau S, Delabarde T, Hackman L. The role of forensic anthropology in disaster victim identification (DVI): recent developments and future prospects. Forensic Sciences Research. 2019 Oct 2;4(4):303-15.

5. Satpathy A, Ranjan R, Priyadarsini S, Gupta S, Mathur P, Mishra M. Diagnostic Imaging Techniques in Oral Diseases. InMedical Imaging Methods 2019 (pp. 59-95). Springer, Singapore.

6. Farman AG, Scarfe WC. Historical perspectives on CBCT. InMaxillofacial Cone Beam Computed Tomography 2018 (pp. 3-11). Springer, Cham.

7. "Galileos Comfort Plus," Dentsply Sirona. [Online]. Available: https://www.dentsplysirona.com/en-gb/explore/imaging-systems/panoramic-cbct/extraoral-3d-units/galileos-comfort-plus. html. [Accessed: 05-May-2020].

8. "CEREC - CAD/CAM for dental practices: Dentsply Sirona," CEREC - CAD/CAM for dental practices | Dentsply Sirona. [Online]. Available: https://www.dentsplysirona.com/en-gb/ explore/cerec.html. [Accessed: 05-May-2020]. 